\title{
Application of failure mode and effects analysis in ecology in Russia
}

\author{
Victoria Borkovskaya ${ }^{1, *}$, and David Passmore ${ }^{2}$ \\ ${ }^{1}$ Moscow State University of Civil Engineering", Yaroslavskoe shosse, 26, 129337, Moscow, Russia \\ ${ }^{2}$ Distinguished Professor, Pennsylvania State University, University Park, PA, 16802, USA
}

\begin{abstract}
The article presents a comprehensive review of the FMEA analysis (the failure modes and effects analysis) in the construction industry in the Russian Federation and suggests the same analysis, which can be applied to ecological issues. The paper analyzes the existing legislative and normative acts in this area, modern approaches to the introduction and regulation of the definition of defects in production and construction. Particular attention is paid to the consideration of Failure Mode and Effects Analysis - this is a formalized procedure for analyzing and finalizing the projected technical facility, the manufacturing process, the operation and storage rules, the maintenance and repair systems of technical facilities, based on the identification of possible (observed) defects with their consequences and cause-effect relationships in their occurrence, and an assessment of the criticality of these defects.
\end{abstract}

\section{Introduction}

One of the main tasks of the quality management system is to ensure the identification of potential inconsistencies (defects) and to prevent their appearance at all stages of the product life cycle. The most important method for solving this problem is to analyze the types and consequences of potential non-conformities (FMEAs). Currently, no less than $80 \%$ of the development of technical products and technologies is carried out using analysis of the types and consequences of potential non-conformities (FMEA-methodology). Analysis of the types and consequences of potential non-conformities is widely used by many world companies both for the development of new designs and technologies, and for the analysis and planning of the quality of production processes and products.

The methodology of FMEA in ecology allows developers to assess risks and possible damage caused by potential inconsistencies in design and technological processes at the earliest stage of designing and creating a finished product or its components. The scope of the method covers all stages of the product life cycle and any technological or business processes. The greatest effect is provided by the application of FMEA at the design and process design stages; however, in the current production the method can be effectively used to eliminate inconsistencies and their causes that were not identified during development or due to factors of variability in production processes.

\footnotetext{
"Corresponding author: BorkovskayaVG@mgsu.ru
} 
Reducing environmental risks at all stages of the product life cycle is relevant for everyone and always, especially where there are special requirements for reliability and safety. In the US in the defense industry, the FMEA technique has been widely used since the early 1940s, in the aerospace industry since the 1960s, and in the automotive industry since the 1970s. In the USSR, the method was available for civilian enterprises from the 1960s. Since the fifties of the last century, no nuclear submarine and no nuclear power plant was designed or built in any country of the world without the application of this simple methodology. In the Russian Federation, the application of the FMEA methodology is required by the industry standards of the QMS of the automotive industry, Russian Railways and Gazprom. The technique is simple, but rarely used correctly [1].

Fundamentals of the FMEA procedure were laid in the 60 s of the twentieth century in the United States. It was first used by the US National Aeronautics and Space Administration (NASA). In the 1980s, the method was further developed and applied in the automotive, aviation, nuclear and other industries of the US industrial production, and then in Europe and Japan. In Germany, the FMEA method is standardized (DIN 25448) [2].

The first information in Russian, devoted to the method of the analysis of species, consequences and criticality of failures (FMESA), appeared in the journal Reliability and Quality Control in the late 1980s. On January 1, 1997, the interstate standard GOST 27.31095 "Reliability in engineering" was introduced. Analysis of species, consequences and criticality of failures. Basic provisions ». The standard was in accordance with the international standard IEC 812 (1985). The Resolution of the Russian Federation (RF) Committee for Standardization, Metrology and Certification GOST 27.310-95 was put into effect directly as the state standard of the Russian Federation on 01.01.1997 [3].

In 1992, the BSI Group published the world's first standard for environmental management systems for organizations - BS 7750. [4] It is noteworthy that this standard did not appear as a result of state activities but on the initiative of British industrialists faced with stricter environmental legislation and the need for adequate managerial decisions. This standard became the basis for the development of the international series of ISO 14000 standards published in 1996 by the International Organization for Standardization ISO. As of 2010, ISO 14001 is currently used by at least 223,149 organizations in 159 countries [ 5 , 6]. OHSAS 18001, Occupational Health and Safety Assessment Series, (officially BS OHSAS 18001) is an internationally applied British Standard for occupational health and safety management systems. It exists to help all kinds of organizations put in place demonstrably sound occupational health and safety performance. It is a widely recognized and popular occupational health and safety management system [7].

Since January 1, 2002 GOST R 51814.2 "Quality system in the automotive industry. Method for analyzing the types and consequences of potential defects" [8].

At present, the Order of the Federal Agency for Technical Regulation and Metrology of December 27, 2007 No. 522-st introduced GOST R 51901.12-2007. This standard is a modified standard IEC 60812: 2006 "Methods for analysis of system reliability." Method for analysis of species and consequences of failures (FMEA)".

Application of FMEA according to GOST R 51901.12-2007 [9].

This standard establishes Failure Mode and Effects Analysis (FMEA). Failure Mode, Effects and Criticality Analysis (FMECA) methods and provides recommendations on how to use them to achieve the stated objectives by:

- performing the necessary stages of analysis;

- identification of relevant terms, assumptions, criticality indicators, types of failures;

- Definition of the basic principles of the analysis;

- use of examples of necessary technological maps or other table forms.

All the general FMEA requirements listed in this standard apply to FMECA, since the latter is an extension of FMEA. 


\section{Materials and Methods}

The basis for applying the analysis of species and consequences of failure (FMEA) or analysis of species, consequences and criticality of failures (FMECA) can be the following:

a) identification of failures that have undesirable consequences for the functioning of the system, for example, termination or significant deterioration of work or impact on the safety of the user;

b) meeting the requirements of the customer established in the contract;

c) improving the reliability or security of the system (for example, by modifying a project or undertaking quality assurance activities);

d) improving the maintainability of the system by identifying areas of risk or inconsistencies with respect to maintainability [10].

In accordance with the above, FMEA (or FMECA) objectives may be the following:

1. compilation of a list of all potentially possible types of defects of a technical object or the process of its production, taking into account both the experience of manufacturing and testing similar facilities, and the experience of real actions and possible personnel errors in the production, operation, maintenance and repair of similar technical objects;

2. determining possible adverse effects from each potential defect, conducting a qualitative analysis of the severity of the consequences, and quantifying their significance;

3. determining the causes of each potential defect and evaluating the frequency of occurrence of each cause in accordance with the proposed design and manufacturing process, as well as in accordance with the expected operating, maintenance and repair conditions;

4. assessing the adequacy of operations in the technological cycle aimed at the prevention of defects in operation, and the adequacy of methods for preventing defects in maintenance and repair;

5. quantitative assessment of the possibility of preventing a defect by means of the provided operations to detect the causes of defects at the stage of manufacturing the facility and the signs of defects at the stage of operation of the facility;

6. the quantitative assessment of the criticality of each defect (with its cause) the risk priority number of the (RPN), and with a high RPN, refine the design and production process, as well as the requirements and operating rules to reduce the criticality of this defect [11].

Based on the goals and objectives, the application of FMEA promotes:

identification of "weaknesses" in design and technological processes; the formation and maintenance of a safety culture; reducing the number of improvements at the stages of manufacturing and selling products; reduction of design time; increasing the effectiveness of management by involving specialists in different areas in the design process; reducing design costs.

The main stages of FMEA in ecology in Russia. The FMAA process is carried out in five stages:

1. preparation for the analysis;

2. Identification of potential CD discrepancies, their consequences and the possibility of detection;

3. risk assessment;

4. identification of corrective / preventive actions;

5. Assessment of the effectiveness of corrective / preventive actions

With FMEA, a preliminary qualitative assessment of the expected (observed) failure rate of various categories of severity in the operation of facilities can be conducted. Estimates are used:

to rank the failures in order of the necessary modifications with the aim of preventing them using matrices relating the "probability of failure [to] the severity of the consequences." 
Taking into account the recommendations given in this matrix, a decision is made on the need for FMEA [12].

Risk assessment. After receiving expert evaluations as to the Severity Occurrence Detection (S O D), the assessment team calculates the risk priority number (RPN). The RPN is an indicator of development risk and is determined by multiplying the rank of significance, occurrence and detection:

$\mathrm{RPN}=\mathrm{SOD}$

RPN can have values from 1 to 1000 .

The maximum $\mathrm{RPN}=10 \times 10 \times 10=1000$ - the worst state

The minimum $\mathrm{RPN}=1 \times 1 \times 1=1$ - best achievable condition.

In the beginning of work on FMEA it is recommended to set the boundary value of the priority number of risk - RPNgr.

The values recommended by GOST P 51814.2 are: $\mathrm{RPNgr}=100-125$. In the future this value can be reduced to $\mathrm{RPNgr}=50-80$. This will reduce the overall criticality of nonconformities. With the RPN> RPNgr, the design or process is being finalized [13].

To carry out corrective / preventive actions, first select inconsistencies with the largest RPN and move in the direction of higher RPNs to the PCRP. In this case, it is necessary to separately consider inconsistencies having ranks of significance / occurrence or detection greater than or equal to 7 .

Despite the resultant RPN, special attention should be given to inconsistencies, the rankings of which are of the greatest importance [14].

Determination of corrective / preventive actions.

Corrective / preventive actions are then developed, and responsibilities and deadlines are determined. The causes of discrepancies with the highest values of the RPN, as well as the largest significance values are eliminated. The goal is to reduce any of the indicators: the significance, probability of occurrence of the probability of detection.

It should be taken into account that:

- the effort of verification / control actions will only reduce the detection rank;

- the possibility of inconsistency can be reduced only by eliminating one or more reasons for the type of non-compliance;

- only revision of the design will reduce the rank of significance.

- Alternatives to action should be considered to address the causes of non-conformities.

The following matters should be quantified:

- the estimated value of the PCR after the introduction of the proposed actions;

- complexity of eliminating causes (full or partial);

- the costs of eliminating causes (full or partial)

- absolute and / or in relation to the costs of eliminating the consequences.

At this poiont, documents necessary for implementation are filed, sent for approval, and then for execution to responsible persons. The completed form is registered. The team is temporarily disbanded.

Assessment of the effectiveness of corrective / preventive actions.

The procedure is to assess the effectiveness of planned corrective / preventive actions. The leading FMEA team monitors the progress of implementation of planned activities and collects data on implementation results.

After the completion of stages 1-5, the host FMEA team collects the team of specialists in the same composition. A second analysis is carried out taking into account the earlier work carried out. If all planned activities are implemented and the effect of corrective / preventive actions is adequate to the goals set for their development, the procedure is completed and the team of specialists is dissolved. In the event that any of the actions did not lead to the planned results, a decision is made to conduct a reanalysis. 


\section{Results and Discussion}

Using FMEA has potential to manage potential problems and issues in ecology in Russia:

- reducing the cost of the product (service) while improving quality (in this case, not only of production but also in the environment);

- a significant reduction in the number of product modifications in the following stages of the production stage;

- reduction of the terms of preparation of production;

- Increase satisfaction in three spheres: commercial and industrial production, harmonization with the environment and pro-environmental interests, and ultimate customer satisfaction with products integrated with environmental benefits.

What is the secret of a simple technique? The answer lies in the effectiveness of the FMEA procedure, which allows, by foreseeing and analyzing possible defects and (or) failures at any stage of the product life cycle, to improve the quality of the design, process or system, to prevent possible defects and reduce the possible consequences from them.

The meaning of FMEA is that, having initially spent a relatively small amount of effort, resources and resources, to get a significant effect, reducing the number of failures and negative consequences from them. By the way about the consequences, the costs from them can be much more than from the failures themselves. For example, the consequences of the destruction of a node or part can be many times greater than the cost of a car, a convoy, a car, a sea or river vessel [15].

Features of FMEA application are practical experience and knowledge of specialists, team approach, brainstorming. It is very important that in the absence or inadequate efficiency of at least one of these components, the methodology does not work. And if we have no problems with experience, then the team approach and brainstorming demand a certain culture and knowledge of certain rules, which is difficult for us in the construction field, where the FMEA method has been actively introduced for 8 years.

DFMEA is the FMEA structure. FMEA design is an analysis procedure of the originally proposed design and its refinement. This will avoid the start-up of untreated construction, improve the design, and provide technological measures to reduce defects. FMEA-analysis of the design can be carried out both for the design being developed and for the existing one. The working group for the analysis usually includes representatives from the departments of development, production planning, sales, quality assurance, and representatives of pilot production [16].

DFMEA is the FEMA structure. FEMA design is an analysis procedure of the originally proposed design and its refinement. This will avoid the start-up of untreated construction, improve the design, and provide technological measures to reduce defects. FMEA-analysis of the design can be carried out both for the design being developed and for the existing one. The working group for the analysis usually includes representatives from the departments of development, production planning, sales, quality assurance, and representatives of pilot production [17]. DFMEA is the FEMA structure. FEMA design is an analysis of the originally proposed design and its refinement. This will prevent the start-up of untreated construction. FMEA-analysis of the design can be carried out both for the design being developed and for the existing one. The working group for the analysis, as well as representatives of the departments of development, production planning, sales, quality assurance, and representatives of pilot product.

Significant results after the introduction of FMEA appear after 12-18 months. Fig. 1 shows a typical cost-benefit ratio from the application of the FMEA methodology. The costs of training and additional hours of work of highly qualified specialists in FMEA-teams are taken into account. 


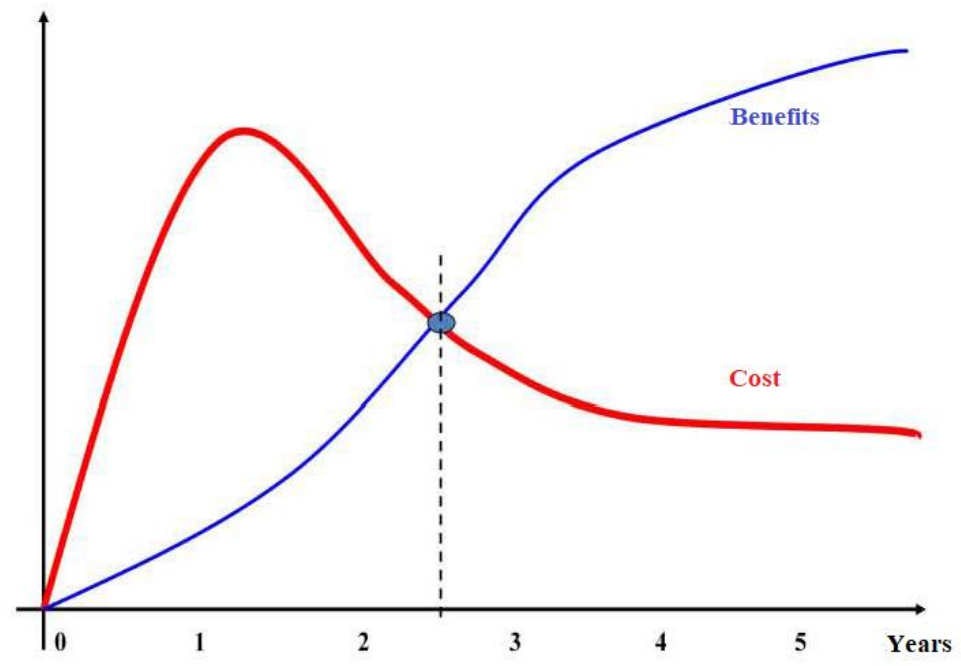

Fig. 1. Cost benefit ratio from FMEA application [18].

Stages of introduction FEMA - the methodology is as follows:

1. Introductory lecture for top managers and chief specialists of the enterprise. In the proposed model, environmental concerns and expertise will be factored into the process.

2. Step-by-step and systematic implementation of FMEA, provision of resources, appointment of responsible, etc.

3. The formation of the required number of multifunctional teams - for various products and technologies being developed. Training for selected participants of FMEA teams is carried out on existing designs and technologies. They know the information about failures, frequencies of one or another reason, etc.

4. Conducting practical exercises on trial sites and technologies.

5. Prototypes of scales of estimations, forms for input of the intermediate and final information can be taken from GOST R 51814.2 [19]. However, you need to creatively reconsider and make them more adapted to the specifics of the enterprise.

6. After the modernization of the scales and forms, the documents should be standardized.

7. The work of FMEA teams should be conducted systematically.

FMEA is an effective method for assessing potential non-conformities and reducing the risk of their occurrence in environmental management systems. The classic FMEA process is adapted to the study of potential environmental risks. The modernized FMEA method was named PNEIA (analysis of potentially hazardous environmental impact). The method is based on real experience of SADACI NV (Belgium), which is a producer of ferromanganese, ferromolybdenum and molybdenum oxides. The PNEIA table is a modernized unified FMEA data registration form. It takes into account the circumstances that may influence the nature and extent of environmental impact among the analyzed aspects. Three scenarios for the development of events are considered: normal (normal) operating conditions; abnormal conditions; emergency situations. FMEA-repair is a modified method of FMEA, applicable in the development of preventive maintenance programs, prioritizing measures and improving the efficiency of equipment.

FMEA-repair, like other FMEAs, should be the result of teamwork. But in this case the team should consist of repair managers, engineers of mechanics. Carrying out appropriate measures to maintain the operability of equipment is not an end in itself [20].

Service repair - service provider, production units - intermediate consumer, and the company as a whole - the end user of services provided by the repair service. 
Objectives of preventive maintenance of equipment: reduction of delays in the delivery of products; reduction of the cost of repair due to reduction of emergency overtime and urgent delivery of spare parts; Increase the average time between failures in work; Reduction of downtime. Along with existing computer programs, FMEA-repair can be used as a simple tool for effective organization of preventive maintenance and repair of equipment, analysis of complex information.

Thus, FME-methodology is used in various spheres of production and services.

The economic benefits of using FMEA in ecology. FMEA promotes a new way of thinking of modern scientific and technical quality assurance [22].

FMEA promotes a new way of thinking of modern scientific and technical quality assurance.

Application of the FMEA method:

- reduces the number of changes introduced (see Fig. 2) at the production stage and the cost of implementing changes (Fig. 3);

- eliminates errors and related defects, and, therefore, eliminates claims, lawsuits and significant costs for the elimination of defects;

- Improves the effectiveness of changes (improvements).

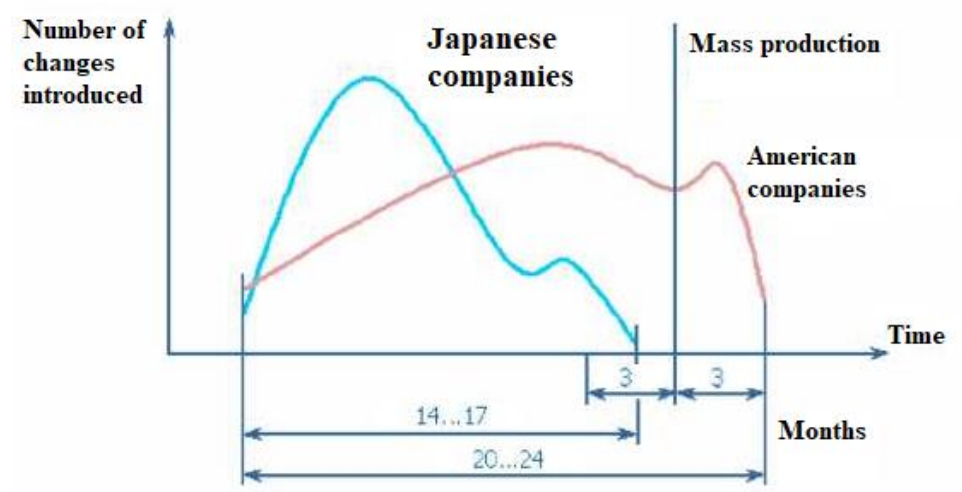

Fig. 2. Amount of time changes introduced by US and Japanese eco-friendly construction companies.

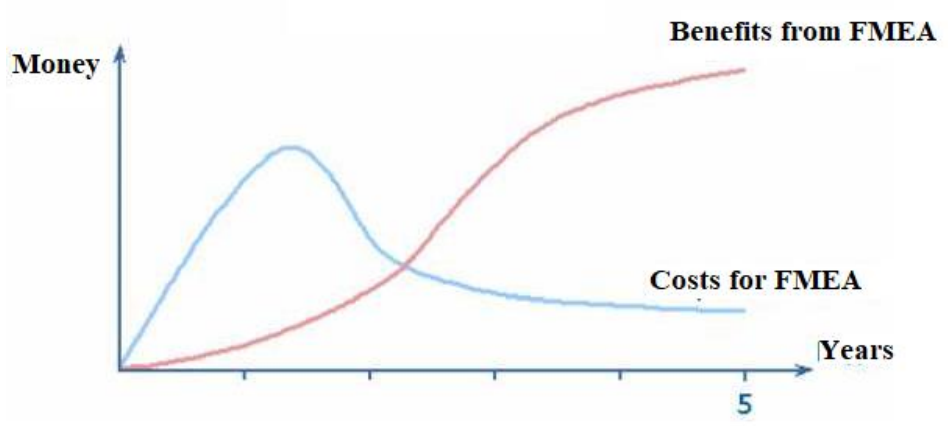

Fig. 3. Benefits from applying FMEA.

The application of the method for analyzing the types and consequences of potential nonconformities is based on the following principles: 
Teamwork. FMEA is conducted by a specially selected multifunctional team of experts. The effectiveness of the analysis directly depends on the professional level, practical experience and coherence of the actions of specialists.

Hierarchy. For complex products, processes and processes of manufacturing complex technical objects, the product / process as a whole, as well as its components (details / operations) are analyzed.

Iterative. The analysis is carried out repeatedly; it is renewed when new factors are identified and any changes that entail a change in the consequences and their risks.

Registration of data. The analysis of the types and consequences of potential nonconformities and its results should be documented

When conducting an FMEA, several rules must be observed:

- integrity in determining points and determining the PCR; if double standards are applied, then the technique loses all meaning;

- the process should be managed by a professional facilitator, who is able to ensure compliance with all conditions and rules;

- at the stage of proposing ideas, it is impossible to criticize, disputes and discussions are canceled;

- at the stage of proposing ideas, their number is more important than quality;

- separation in time of the process of generating ideas and the process of their evaluation;

- follow the rules of brainstorming, not to leave the topic, not to recall each other's past grievances and try not to scream (although this is still difficult);

- ideas are brief, all ideas are written down;

- at the discussion stage, deliberately "absurd" ideas are discarded;

- leave protocols, drafts and all additional materials, this will ensure the accumulation of experience, even with the staff turnover that is in the enterprises;

- the developed measures and the timing of their implementation should be coordinated with all interested services and issued under the signature of the authorized manager for compulsory execution;

- the course of implementation of measures is monitored not only by the final date of their implementation, but also in the process of work.

Despite the fact that the FMEA technique is relatively "simple" in comparison with other special QMS methods, however, FMEA has several "deadly" diseases:

- a formal approach;

- misunderstanding of the specifics of application at different stages of the product life cycle;

- lack of understanding of the relationship with other QMS methods;

- Failure to understand the specifics and application of the FMEA system, design, process and product;

- Lack of support from senior management.

Despite the simplicity and effectiveness of the FMEA methodology, it must not be forgotten that it has one minus. Where the causes of potential defects lie particularly "deep," the technique may not yield results. In this case, more complex techniques, such as DOE ("Experiment Planning"), are needed. But in this case it is necessary that the company has one or more specialists trained in DOE methods.

The correct application of the FMEA technique will avoid unnecessary losses in the finalization of the design and process. The rule of tenfold costs has not been canceled yet, and any error noted in time is sometimes a saving of hundreds of thousands of rubles, not to mention reducing risks to the end user. An example of Western enterprises suggests that the use of FMEA allows not only to reduce defects, but, if necessary, to justify their innocence in disputable situations. 
When implementing the FMEA, try to learn the experience of advanced Russian, joint and foreign enterprises (even Eastern European ones such as Czech Republic, Slovenia, Poland). This will allow us to better understand the application of the methodology at various stages of the product life cycle and the need to apply FMEA in conjunction with other QMS methods that are intertwined and simply inseparable. And most importantly, it is not a onetime use of the methodology, but the application of the FMEA concept, similar to the one that exists in procedures for planning the quality of APQP and ANPQP prospective products, when the FMEA methodology is systematically and consistently applied at all stages of production preparation.

\section{Conclusions}

The costs of eliminating the consequences of a failure (defect) directly depend on the time of detection. The sooner a failure is discovered, the less it will cost to fix it. If a failure is detected in the later stages of development or launch of a product, its impact becomes much more palpable. In the case of environmental damage, this damage is much harder to reverse after it occurs.

FMEA for ecological process is one of the tools used to detect possible defects or failures at the earliest stage: the development of a product or process. Detection of a failure at an early stage of product development (PD) using FMEA provides the following advantages:

Several options for reducing risks;

- Higher verification capability;

- Appearance of "interaction" between the Design and the Process, according to which this Construction is made;

- Improved Construction to optimize the final assembly;

- The use of cheaper solutions, compared with the subsequent stages of the life cycle of the product (LCP).

Thus, this methodology is effective in identifying and correcting process failures at the earliest possible stage, thus avoiding the severe consequences of a low level of productivity.

In Russia environmental process for FMEA-analysis is represented by several standards: ISO14001, OHSAS 18001, GOST R 51814.2 and P 51901.12. The very methodology of the analysis of failures and errors is used quite rarely, a greater preference is given to the creation of "risk matrices". This approach is more comprehensive, but also more financially costly.

\section{References}

1. A. Zinatullin, E. Chibisova, Strategy of sustainable development of regions in Russia 5, 324-328 (2011)

2. D. Rodionov, A. Afanasov, A. Gorova, World of economy and law 4-5, 18-30 (2014)

3. V.G. Borkovskaya, Applied Mechanics and Materials. Engineering Management 475476, 1703-1706 (2013) DOI: 10.4028/www.scientific.net/AMM. 475-476.1703

4. H. Skipper, J. Kwon, Risk Management and Insurance (Perspectives in a Global Economy, Wiley, 2007)

5. D. Kahneman, A. Tversky, Prospect Theory: An Analysis of Decision under Risk 47(2), 263-291 (1979)

6. V.G. Borkovskaya, Applied Mechanics and Materials. Materials Science and Mechanical Engineering. Chapter 2: Building Materials and Construction Technologies 1, 287-290 (2013) DOI: 10.4028/www.scientific.net/AMM.467.287 
7. R.K. Sawyer, The Cambridge Handbook of the Learning Sciences (Cambridge University Press., 2006)

8. V.G. Borkovskaya, Advanced Materials Research. Engineering Education 860-863, 3009-3012 (2013) DOI: 10.4028/www.scientific.net/AMR. 860-863.3009

9. V.G. Borkovskaya, Manufacturing Management and Engineering Management 945949, 3012-3015 (2014) DOI: 10.4028/www.scientific.net/AMR.945-949.3012

10. D. Bandaly, L. Shanker, Y. Kahyaoglu, A. Satir, Risk Management 15(1), 1-31 (2013) DOI:10.1057/rm.2012.8

11. Y. Lin, R.D. MacMinn, R. Tian, J. Yu, Journal of Risk and Insurance 84(S1), 345-365 (2017) DOI:10.1111/jori.12196

12. D. Passmore, R.M. Baker, Research in organizations: Foundations and methods of inquiry (Berrett-Koehler, San Francisco, 2005)

13. S. Stranieri, L. Orsi, A. Banterle, Supply Chain Management: An International Journal 22(2), 145-159 (2017) DOI:10.1108/SCM-07-2016-0268

14. J. Lathrop, B. Ezell, Safety Science 99(B), 187-195 (2017) ISSN 0925-7535, DOI:10.1016/j.ssci.2017.04.006

15. V.G. Borkovskaya, AMM. Chapter 2: Building Materials and Construction Technologies, 287-290 (2013) DOI: 10.4028/www.scientific.net/AMM.467.287 